\section{The 32nd Annual Meeting of the J.B. Johnston Club for Evolutionary Neuroscience and the 24th Annual Karger Workshop in Evolutionary Neuroscience}

The 2012 meeting of the J.B. Johnston Club for Evolutionary Neuroscience and the Karger Workshop in Evolutionary Neuroscience will be held immediately before the annual meeting of the Society for Neuroscience on Thursday, October 11 (the Karger Workshop), and Friday, October 12 (the regular JBJC meeting), 2012. Both meetings will take place at the Bourbon Orleans Hotel in New Orleans, La., USA.

This year's Karger Workshop, made possible by the continuing support of Karger Press, was organized by Leo Demski and is entitled 'Evolution of Brain Complexity and Animal Minds'. The Workshop features six invited talks by speakers who will review brain-behavior relationships in octopus, various fishes, reptiles, birds and mammals. The Workshop, dedicated to the memory of Ted Bullock, aims to identify neurobiological specializations that make the behavior of individuals within these groups unusually complex. On the following day, the program for annual JBJC meeting will consist of 15 talks submitted by JBJC members selected by the JBJC Program Committee (Ann Butler, Barbara Finlay, and Michael Pritz) plus a presentation by this year's invited Karger Speaker, Dr. Binyamin Hochner. Additional information and the final schedule of talks will be mailed to JBJC members before the meeting, and will be posted on the JBJC web site (www. jbjclub.org).

2012 Karger Workshop in Evolutionary Neuroscience: Evolution of Brain Complexity and Animal Minds

\section{Organized by: Leo S. Demski (New College of \\ Florida, Sarasota, Fla., USA)}

Speakers giving presentations at the 2012 Karger Workshop in Evolutionary Neuroscience are listed below. The final schedule of talks will be sent to the membership prior to the meeting and will be available at the registration desk during the meeting.

\section{Relationships between Brain and Behavioral Complexity in Insects} (USA)

Sarah M. Farris, Morgantown University, Morgantown, W. Va.

\section{The Brain/Body/Behavior Organization in an Animal with an Unusual Morphology - An 'Embodied' View on the Organization of the Nervous System of Octopus vulgaris}

Binyamin Hochner, Hebrew University, Jerusalem (Israel)

'Brainy' Fishes: Considerations of Neural Complexity and Mental Diversity

Leo S. Demski, New College of Florida, Sarasota, Fla. (USA)

\section{Variation in Reptilian Brains and Cognition}

R. Glenn Northcutt, University of California at San Diego, San Diego, Calif. (USA)

\section{What Is It Like to Be a Bird? Insights from Avian Brains and Avian Behavior}

Georg F. Striedter, University of California at Irvine, Irvine, Calif. (USA)

\section{Three Evolutionary Trajectories for Evolving Large \\ Brains in Humans, Elephants and Cetaceans: \\ Similarities and Differences in the over 700-Gram \\ Club}

Paul R. Manger, University of the Witwatersrand, Johannesburg (South Africa)

\section{J.B. Johnston Club for Evolutionary Neuroscience Meeting Abstracts}

Abstracts for talks scheduled for the 2012 annual meeting of the J.B. Johnston Club for Evolutionary Neuroscience are listed in alphabetical order by presenting author. The final schedule of talks will be sent to the membership prior to the meeting and will be available at the registration desk during the meeting. This

\section{KARGER}

Fax +41613061234

E-Mail karger@karger.ch

www.karger.com (c) 2012 S. Karger AG, Basel

0006-8977/12/0801-0064\$38.00/0

Accessible online at: www.karger.com/bbe 
year's Karger Invited Guest will be Dr. Binyamin Hochner. The title of his talk will be: 'Conservation, convergence and variability in the neurophysiological organization of a learning and memory system in advanced Molluscan Cephalopods - octopus and cuttlefish'.

\section{A Web-Accessible Multimodal Atlas of the Brain of the Bottlenose Dolphin (Tursiops truncates)}

\section{Jacapo Annese}

The Brain Observatory, University of California San Diego,

San Diego, Calif., USA

(jannese@ucsd.edu)

Historically, the brains of cetaceans have been the subject of anatomical investigations seeking the structural determinants of their behavioral specializations, such as echolocation, communication, and intelligence. Presently, an intense debate is underway on whether dolphins should be granted the status of 'persons' on account of their purported human-like cognitive functioning. The ethical, legal, and socio-economic implications of this debate are substantial, because a resolution would affect whaling, captivity, and the use of dolphins in entertainment. Many of the arguments for and against treating dolphins as 'non-human persons' are based on different interpretations of neuroanatomical evidence; in reality, this evidence is extremely scarce and disorganized. The lack of a substantial body of knowledge on the functional architecture of the brain of dolphins and other marine mammals is due to the rarity of specimens and the very limited access to the few existing collections. Even in those cases where histological material has been studied, the examination was limited to very small tissue samples that only show local morphological features. What is missing in the field of cetacean neuroanatomy is a comprehensive survey at the system level that can provide comparative anatomical data from multiple imaging modalities and accessible at multiple levels of resolution.

We have created a web-based neuroimaging resource for the brain of the dolphin that will be accessible to researchers, scholars, and educators world-wide. The web-based multimodal atlas contain 3-dimensional (3D) image data from postmortem Magnetic Resonance Imaging (MRI), as well as surface-based models of individually-labeled structures. Radiologic images are correlated to series of giant histological sections acquired serially through the whole brain. These images are accessible via the web in the form of terapixel files representing the whole sections at $20 \times$ magnification. The data is displayed using open source code originally developed and then distributed by Google. Understanding how the dolphin brain leverages on typically mammalian, yet uniquely specialized and arranged components, is one of the great challenges of comparative neurobiology. The web-accessible atlas is a significant step towards achieving a comprehensive neuroanatomical database to support rigorous quantitative comparative studies of the cetacean brain. The information contained in the images and metadata will be crucial to validate or demystify current hypotheses on cognitive abilities of cetaceans, and even elucidate, by comparison, important mechanisms underlying the functional architecture of the human brain.

\section{Cytoarchitectonic Characteristics of the Cerebral Cortex of the Nine-Banded Armadillo (Dasypus novemtinctus)}

\author{
Pooja Balaram ${ }^{a}$, Roger L. Reep ${ }^{b}$, Jon H. Kaas ${ }^{a}$ \\ aDepartment of Psychology, Vanderbilt University, Nashville, \\ Tenn., ' Department of Physiological Sciences, University of \\ Florida, Gainesville, Fla., USA \\ (pooja.balaram@vanderbilt.edu)
}

We have examined the cortical organization of the cerebral hemispheres of the nine-banded armadillo using modern immunohistochemical techniques and a battery of markers that are known to identify cortical areas in other mammalian species. Very little is known about brain organization in armadillos, which are eutherian mammals of the superorder Xenarthra that includes sloths, anteaters, and armadillos. While the brain of the nine-banded armadillo is relatively small compared to its body size, the cerebral hemispheres are dominated by a proportionately large piriform cortex, with a number of shallow fissures and expansive fiber tracts that originate from the large olfactory bulb. These structures subserve the armadillos' use of olfaction in finding food. In addition, the hippocampus is relatively large in the nine-banded armadillo, suggesting that armadillos have good memories for locations of multiple food sources and burrows. The neocortex of the nine-banded armadillo occupies a relatively small portion of the forebrain, but contains distinct primary visual, auditory, and somatosensory areas. These areas are usually characterized by discrete lamination and a pronounced layer 4, which are identifiable in Nissl and myelin preparations. Staining for vesicular glutamate transporter 2 (VGLUT2) also identifies primary sensory areas in rodents and primates, as it delineates thalamocortical terminations in cortex from sensory nuclei. Secondary sensory areas such as S2 and V2 also likely exist adjacent to primary sensory areas, but are less distinct in histological preparations. Retrosplenial areas and areas of frontal cortex were also identifiable by their characteristic architectonic appearances. A frontal agranular region likely corresponds to primary motor cortex, which is usually identifiable by staining for SMI32, a marker that labels non-phosphorylated neurofilament protein in neurons and thus, visualizes the large axons of corticospinal neurons in mammalian motor cortex. Comparisons of cortical organization in other members of the major branches in the radiation of placental eutherian mammals indicates that eutherian mammals retained a collection of 10 or more cortical areas from early mammalian ancestors, while adding a separate primary motor area and perhaps other areas as well as a corpus callosum. By comparing the anatomical boundaries defined by our battery of immunohistochemical preparations, we have generated an area map of the architectonic organization of the neocortex in the nine-banded armadillo brain. 


\section{Perspectives on the Evolution of Complex Brains and Behaviors in Cephalopods}

Jennifer A. Basila, Frank W. Grasso ${ }^{b}$

Departments of aBiology and ' ${ }^{\text {P }}$ sychology, Brooklyn College,

The City University of New York, New York, N.Y., USA

(jbasil@brooklyn.cuny.edu)

Cephalopods are an ancient group of marine molluscs $(\sim 850$ extant species) with complex brain organization and correspondingly sophisticated behavioral repertoires. Predatory species of cephalopods, extinct and extant, competed and compete with marine vertebrate taxa because they often occupy similar ecological niches. Convergent processes may thus be acting (and may have acted) upon both groups and are largely unexplored. A major division among extant species falls between those retaining external shells (the slow, scavenging Nautiloids), considered ancestral, and those with reduced or absent shells (the fast, predatory Coleoids), a more recent modification. Brain complexity follows similar lines: the nautiloid brain is considered plesiomorphic, while coleoid brains are more derived [Shigeno et al., 2008]. Evidence supports that the radiation of coleoids from the Devonian until present was driven, in part, by mutual competition between marine vertebrates and cephalopods - shaping body forms and behaviors ['a cognitive radiation'; Packard, 1972; Aronson, 1991]. Specifically, fast locomotory capabilities in coleoids coincide with the advent of efficient swimming styles in bony fishes [Chamberlain, 1993]. We posited that the foundations of coleoid brain complexity have earlier origins - from competition with diverse cephalopod groups and other marine forms before the advent of the bony fishes [Grasso and Basil, 2009]. Extant nautiloids have simpler brains than coleoids: 13 lobes versus $\sim 40$ lobes [Young, 1971]. However the nautiloid brain is large and complex in comparison to other molluscs and possesses higherorder centers. Brain expansion coincident with the coleoid radiation may be built upon an ancient architecture that predates the advent of bony fishes. Indeed, the learning and memory capabilities of nautiluses share marked similarities with the coleoids in capacity, duration and flexibility [Grasso and Basil, 2009]. In addition, the transition from a diffuse brain to an encephalized brain occurred 3-4 times in the evolution of molluscs, independent of the cephalopod lineage [Kocot et al., 2011]. Taken together, these lines of evidence point to an evolutionary scenario where coleoid brain complexity resulted from at least two phases of interspecifc completion, at least one before and one after the advent of bony fishes.

\section{References}

Aronson RB (1991) Ecology, paleobiology and evolutionary constraint in the octopus. Bull Marine Sci 491:245-255.

Chamberlain JA (1993) Locomotion in ancient seas: Constraint and opportunity in Cephalopod adaptive design. Geobios 26(suppl 1):49-61.

Grasso FW, Basil J (2009) The evolution of flexible behavioral repertoires in cephalopod molluscs. Brain Behav Evol 74:231-245.

Kocot KM, Cannon JT, Todt C, Citarella MR, Kohn AB, Meyer A, et al (2011) Phylogenomics reveals deep molluscan relationships. Nature 477:452-456.

Packard A (1972) Cephalopods and fish: the limits of convergence. Biol Rev 47:241-307.
Shigeno S, Sasaki T, Moritaki T, Kasugai T, Vecchione M, Agata K (2008) Evolution of the cephalopod head complex by assembly of multiple molluscan body parts: Evidence from Nautilus embryonic development. J Morphol 269:1-17.

Young JZ (1971) The Anatomy of the Nervous System of Octopus Vulgaris. Oxford, Clarendon Press.

\section{Auditory Specialization within the Cichlidae: Morphological Specializations and Performance Enhancements in Malagasy South-Asian Cichlids}

\author{
C.B. Braun ${ }^{a-c}$, Z. Baldwin', J.S. Sparks ${ }^{c}$
}

aPsychology, Hunter College, ${ }^{\mathrm{b}}$ Graduate Center, City University of New York, 'Department of Ichthyology, American Museum of Natural History, New York, N.Y., USA

(cbraun@hunter.cuny.edu)

The primitive sense of fish hearing relies on inertial displacement of otoliths for the detection of low frequency sound, primarily in the acoustic nearfield. The evolution of novel pressuretransduction mechanisms enhances the hearing ability in descendant taxa, increasing sensitivity at all frequencies and increasing the upper frequency limit of hearing. This increase of sensitivity and bandwidth enhances communication at higher frequencies, particularly in shallow waters and very likely increases the distance range of audition generally. Unsurprisingly, these advantages have been exploited numerous times in vertebrate evolution. In teleost fish alone, novel transduction mechanisms have evolved several times, in as many as 20 evolutionarily independent lineages. We describe one case of auditory specialization within the Cichlidae. Cichlids display a wide range of gas bladder morphology, but in the Malagasy and South-Asian lineages, a diversity of morphologies have arisen, many of which bring the gas bladder into close contact with the otolithic inner ear. Using CT and MRI imaging, we have described the gas bladder and its relationship to the caudal neurocranium in representatives of all cichlid clades. With the Malagasy and South-Asian lineages, two evolutionarily distinct trends are apparent. In Ptychochromines, rostral gas bladder extensions approach and sometimes contact the back of the skull, which has variably reduced exocciptal bone thickness. In extreme cases, the gas bladder is nestled into a thinned exocciptal foramina, but does not penetrate the skull. In Etroplines, there is also a range of rostral gas bladder morphologies, which includes multiple anterior chambers of the gas bladder with a range of constrictions and swellings. In many cases, the diverticula continue through the exoccipital foramina and form a large intracranial bulla that are associated with both the saccular and lagenar otolithic organs. All of these morphologies provide some hearing enhancement, with acoustic evoked potential thresholds ranging from highly sensitive (similar to otophysans) to typical cichlid sensitivity (similar to unmodified cichlids such as Astatotilapia burtoni). We will also present ecological data from the type locality of several Malagasy cichlid species, demonstrating that they are found in a range of acoustic niches. 


\section{Internasal Intensity Cues Provide Directional Sniffing Information}

\section{Kenneth Catania}

Department of Biological Sciences, Vanderbilt University, Nashville, Tenn., USA

(ken.catania@vanderbilt.edu)

Eastern American moles demonstrate a remarkable ability to navigate rapidly and accurately to the location of an odor source (under either full spectrum or infrared illumination). This behavior was documented using high-speed video and a pressure monitor to determine the timing of each sniff. Results reveal a sniff cycle that is closely coordinated with nose movements, such that each olfactory sample is taken at a different spatial location. This suggests that serial sampling within an olfactory gradient provides an important cue for stimulus location. But blocking one nostril resulted in localization errors in the direction of the open nostril. In contrast, artificially increasing the odor concentration in a nostril by extending it with a small polyethylene tube (to be closer to the stimulus) resulted in errors in the direction of the tube. These results suggest that both serial sampling and internasal intensity differences during each sniff provide directional information during olfactory localization. The effect of a blocked or extended nostril was primarily apparent at short distances from the stimulus, suggesting that internasal intensity cues are only available close to the stimulus, where the intensity gradient of diffusing odorants is steep.

\section{Patterning of Superfast Motor Behaviors - A Lesson from Vocalizing Fish}

Boris P. Chagnaud

Department of Biology II, Ludwig-Maximilians-University Munich, Martinsried, Germany

(b.chagnaud@gmail.com)

Vocalizations are widely used in vertebrate social communication and are controlled by an evolutionarily conserved hindbrain neural network. Recent comparative evidence suggests that this hindbrain network originated in fish, making them an excellent candidate to investigate the basic properties of vocal pattern generation. One mechanism by which fish produce vocalizations is by rapidly contracting 'superfast' muscles attached to the swim bladder. Fast, repetitive and synchronous activation of all muscle fibers is a prerequisite for generating a single audible sound pulse within the fish hearing range $(>70 \mathrm{~Hz})$. Using intracellular in vivo recordings coupled with extracellular motor output recordings, we investigated the hindbrain central pattern generator (CPG) that leads to highly stereotyped, rhythmic sound production in midshipman fish. The CPG has three nuclei, each with distinct anatomical and physiological properties coding an equally distinct attribute of a muscle contraction series that directly determines temporal call properties. Muscle contraction/ call duration is encoded by a sustained membrane depolarization in vocal pre- pacemaker neurons that receive direct midbrain input. Pacemaker neurons, directly innervated by vocal pre-pacemakers, encode muscle contraction/call frequency by rhythmic, ultrafast oscillations in their membrane potential. Pacemaker neurons, in turn, densely innervate vocal motoneurons whose differential recruitment encodes muscle contraction/call amplitude. Motoneurons, the determinative output stage controlling muscle contraction and hence natural call attributes, show several adaptations for generating the highly synchronous output required for superfast muscle activation. These include (1) a lack of spontaneous activity that could interfere with the rhythmic activation of the entire network, (2) a low excitability leading to a minimum of activation needed across the entire network to generate a muscle contraction, (3) a prominent after-hyperpolarization that prevents tonic firing, (4) GABAergic inhibitory input involved in hyperpolarizing the membrane potential, and (5) extensive gap junctional coupling that leads to shared inputs across the entire motoneuron population. Taken together, motoneuronal intrinsic and network properties translate premotor inputs into a coincident and extremely synchronous, but variable amplitude, output code that leads to synchronous activation of muscles fibers across paired superfast muscles. We propose an analogous suite of motoneuronal properties in other superfast muscle systems similarly dependent on synchronous activation in amphibians, reptiles, birds and mammals [This work was conducted in collaboration with A. Bass, R. Baker and M. Zee].

\section{Hydrodynamic Stimuli Detection by Florida Manatees (Trichechus manatus latirostris)}

\author{
Joseph C. Gaspard III, ${ }^{a}$, Roger L. Reep ${ }^{a}$, Gordon B. Bauer b, c, \\ Kimberly Dziuk ${ }^{b}$, Katharine Nicolaisen ${ }^{b}$, Laura Denum ${ }^{b}$, \\ David A. Mann ${ }^{b, d}$ \\ ${ }^{a}$ College of Veterinary Medicine - Physiological Sciences, \\ University of Florida, Gainesville, Fla., ${ }^{b}$ Mote Marine Laboratory \\ \& Aquarium, Sarasota, Fla., 'New College of Florida, Sarasota, \\ Fla., ${ }^{\mathrm{d} C o l l e g e}$ of Marine Science, University of South Florida, \\ St. Petersburg, Fla., USA \\ (jgaspard@mote.org)
}

Manatees possess specialized sensory hairs (vibrissae) on the face and across the body, a unique arrangement among mammals. Previous research has demonstrated that manatees have good hearing yet poor visual acuity. The anatomical distinctiveness of the vibrissae highlighted by the likely association with aggregations of neurons in the cortex, similar to the barrel cortices in rodents, suggests that somatosensation may be the manatees' primary sensory system. Two Florida manatees were tested in a go/ no-go procedure using a staircase method to assess their ability to detect water movement. Hydrodynamic vibrations were created by a sinusoidally oscillating sphere that generated a dipole field at frequencies 5-200 Hz, which are below the functional hearing limit of the manatee. The manatees detected particle displacement down to 1 nanometer using their facial vibrissae. Testing of the post-facial vibrissae demonstrated that the thresholds 
were slightly elevated in comparison, yet still within an order of magnitude. In order to elucidate the roles of these hairs and how manatees utilize them, a variety of manipulations were conducted which included various degrees of restriction and immobilization of the vibrissae. The results emphasized the critical role of the hairs in the detection of the water movement. The vibrissae of the manatee may act as a three-dimensional array for orientation and navigation, analogous to the lateral line system of a fish.

\section{To Swim or To Crawl: The Effectiveness of Different Locomotor States on Prey Localization}

\author{
Cynthia M. Harley ${ }^{a, b}$, Matthew Rossi ${ }^{b}$, Javier Cienfuegos ${ }^{b}$, \\ Daniel A. Wagenaar ${ }^{b}$, Karen A. Mesce ${ }^{a}$ \\ aDepartment of Entomology, University of Minnesota, St Paul, \\ Minn., ${ }^{b}$ Division of Biology, California Institute of Technology, \\ Pasadena, Calif., USA \\ (cmharley@umn.edu)
}

Medicinal leeches, like many aquatic animals, use water disturbances to localize their prey. This task requires that they are able to separate wave disturbances of prey from those created by other sources. In a previous study we determined that leeches perform this separation based on the frequency and amplitude of water waves.

Two sensory systems guide the leech's prey localization behavior: a distributed mechanosensory system comprising an array of nearly 300 receptors sensitive to water movement, and a visual system comprising 10 simple eyes in the head and an array of visual sensilla that are colocalized with the mechanoreceptors. However, knowing what information is used to direct the leech is only part of the story- we must also know how that information is used.

The seemingly simple task of reaching the prey itself requires that the leech move within the same water which it is using to sense prey. We found that leeches do not localize prey ballistically. Instead, they require continual sensory information to track their prey. Furthermore, in the event that the prey moves, leeches will update their movement direction to respond to the new prey location.

While it would appear that leeches need to continually sense water disturbances to update their percept of prey location, their own behavior is discontinuous - approaching prey involves switching between swimming, crawling, and non-locomotion. Each of these behaviors may allow for different sensory capabilities and may require different sensory filters. Here, we examined the sensory capabilities of leeches during each of these behaviors. We found that while one could expect the non-locomotor phases to direct subsequent behaviors, crawling was unexpectedly more effective for prey localization. During crawling bouts, leeches continually updated their movement direction, heading toward the stimulus. This continual updating was not observed during swimming. Furthermore, in the presence of prey-like stimuli, leeches crawled more often and for longer periods of time.

We did observe, however, a searching-like behavior during some non-locomotor bouts. We found that this behavior was used to orient the leech toward stimuli and over the course of the trial became localized to the same vicinity as the stimulus. However, searching behavior did not occur more often or for a longer duration when prey-like stimuli were present. This idea is consistent with the idea that leeches may utilize an intermittent and random search strategy to maximize their prey encounter rates.

\section{Distribution of Neurons across the Surface of the Mouse Cerebral Cortex}

\author{
Suzana Herculano-Houzel ${ }^{a}$, Charles Watson ${ }^{b, c}$, George Paxinos ${ }^{c, d}$ \\ aUniversidade Federal do Rio de Janeiro, Rio de Janeiro, Brazil; \\ ${ }^{b}$ Curtin University, Perth, ${ }^{C}$ Neuroscience Research Australia, \\ Sydney, dUniversity of South Wales, Sydney, Australia \\ (suzanahh@gmail.com)
}

The mammalian cerebral cortex has traditionally been considered a homogeneous structure, with a constant number of neurons per surface area (N/A) across both cortical areas and species [with the exception of primary visual cortex; Rockel et al., 1980]. However, N/A is neither homogeneous across primate species [Herculano-Houzel et al., 2008] nor across the cortical surface [Collins et al., 2010]. Here we use the isotropic fractionator [Herculano-Houzel and Lent, 2005] to analyze the distribution of neurons across 18 areas of the isocortex of four C57Bl/6J mice. Areas were defined anatomically in 200 micron coronal sections by comparison with the corresponding Franklin and Paxinos [1997] atlas plates and pooled for counting.

Remarkably, the somatosensory areas together contain $25 \%$ of all cortical neurons. The face area of S1 is the single functional area with the most neurons (13.4\% of all cortical neurons), followed by motor cortex ( $10.1 \%$ of all cortical neurons) and primary visual cortex (9.4\% of all cortical neurons). The distribution of neurons across functional areas does not accompany the distribution of surface area; as a result, we find that N/A varies 4.5 -fold across functional areas.

Strikingly, the distribution of neurons mirrors closely the distribution of cortical volumes across the functional areas, and indeed local cortical volumes vary as a linear function of local numbers of neurons, with the exception of the primary visual areas, which hold more neurons than expected for their volume, with neuronal densities that are twice those found in other areas. Neuronal density does not vary systematically across the non-visual areas. Interestingly, surface area is a different function of number of neurons across sensory (exponent $0.867, \mathrm{p}=0.0030$, including visual areas) and non-sensory functional areas (exponent 0.914 , $\mathrm{p}=0.0001$ ); and cortical thickness increases with local neuronal density, but as different functions across dorsal-anterior, dorsalposterior, and medial/lateral areas. In contrast, the distribution of other cells mirrors closely the distribution of cortical volume across all areas, including the visual cortex.

These findings suggest that neurons are allocated to functional areas (except for visual areas) on the basis of a common relationship between number of neurons and average neuronal size, leading to cortical volumes that are a shared function of numbers of neurons; but neurons are distributed differently between sensory 
and non-sensory areas in regard to surface area. Rather than a continuous gradient of neuronal densities across the cortical surface, then, our data suggest a discrete discontinuity in the distribution of neurons between visual and all other non-visual cortical areas.

\section{References}

Collins CE, Airey DC, Young NA, Leitch DB, Kaas JH (2010) Neuron densities vary across and within cortical areas in primates. Proc Natl Acad Sci USA 107:15927-15932.

Franklin KBL, Paxinos G (1997) The Mouse Brain in Stereotaxic Coordinates. San Diego, Academic Press.

Herculano-Houzel S, Collins CE, Wong P, Kaas JH, Lent R (2008) The basic non-uniformity of the cerebral cortex. Proc Natl Acad Sci USA 105:12593-12598.

Herculano-Houzel S, Lent R (2005) Isotropic fractionator: a simple, rapid method for the quantification of total cell and neuron numbers in the brain. J Neurosci 25:2518-2521.

Rockel AJ, Hiorns RW, Powell TP (1980) The basic uniformity in structure of the neocortex. Brain 103:221-244.

\section{Dualism, Monism, Proxy Data, and Argument by Analogy in the Study of Comparative Animal Consciousness and Cognition}

Louis N. Irwin

Department of Biological Sciences, University of Texas at El Paso, El Paso, Tex., USA

(lirwin@utep.edu)

Dualism - a philosophical position antithetical to both Idealism (the world is purely perception) and Materialism (the world is purely substance) - has persisted since ancient times to frame the issue of animal consciousness and cognition. In modern times, the presumption of evolutionary continuity and arguments by analogy have generally favored the monistic view that consciousness and cognition are based on biological traits that grade nonlinearly across phylogenetic lines. Not everyone agrees; views that consciousness is a purely human feature, or restricted to mammals and birds, or that non-human animals are incapable of a 'theory of mind' are not uncommon. Lloyd Morgan's Canon never assume more complex explanations for animal behavior when simpler explanations will suffice - has dampened scientific consideration of animal consciousness and cognition for a century. Arguments by analogy for animal consciousness appear to work for taxa with a common ancestry, but are problematic across large phylogenetic divides. Ecological adaptation theory has advanced the comparative study of animal cognition, but is challenged by experimental difficulties. Just as behaviorism (despite its faults) circumvented the philosophical obstacles to the scientific study of animal behavior in the early 20th century, the time may have come to set aside philosophical debate on the animal mind, in favor of resort to proxy data for animal consciousness and cognition. The use of directed attention will briefly be described as one such example.

\section{To What Extent Do Glial Invariance and Variable Neuron Mass Explain the Distinct Scaling Rules for the Cortical Shape and Composition of Different Mammalian Orders?}

\section{Bruno Mota, Suzana Herculano-Houzel}

Instituto de Ciências Biomédicas, Universidade Federal do Rio de Janeiro, Rio de Janeiro, Brazil

(wronski@gmail.com)

Studies of the comparative neuroanatomy have shown that, across different mammalian orders, quantities such as cortical mass, number of neurons, number of glia, grey and white matter volume and shape can be pairwise related to each other by simple power law scaling rules, strongly suggesting that cerebral structures are built by emergent scale-invariant mechanisms. In general the exponents of such scaling rules vary considerably from order to order. However, and relevantly to what follows, it turns out that the number of non-neuronal cells scale almost identically with structure mass across different orders and cerebral structures.

Furthermore, using a few simple but plausible hypotheses, it is possible to derive from these measurements the power law scalings of quantities that are not directly measurable, such as average WM axonal length and caliber, and average neuronal and glial masses. The latter two quantities in particular tell an interesting story. Average cortical glia masses vary little across species, suggesting glia are largely invariant in size (and, presumably, function), each glial cell being associated with a nearly fixed quantum of neuronal matter. Average cortical neuronal cell mass, on the other hand, varies by nearly two orders of magnitude across different species, typically increasing with structure mass, but at different rates for different orders. So, while neurons in primate cortices change average sizes only modestly despite the wide difference in cortical size, in rodents neurons become on average much larger as cortical mass increases. As a result, a large rodent cortex will have far fewer neurons than a primate brain of approximately the same size.

It may be worthwhile to try to understand this difference in neuron-scaling strategies in terms of diverse evolutionary pressure and phylogeny leading to the selection of different cortical physiologies; but what we do in what follows is invert the question and ask if the scaling rules that have been obtained relating measured and estimated quantities for different orders can be explained solely (or at least with the fewest further assumptions) by this discrepancy in neuronal mass scaling alone. We thus construct a simple model for the cortex with identical assumptions pertaining to different orders, except for the distinct average neuronal mass scaling rules, and show that it is indeed possible within this simple framework to extract therein the observed scaling rules. 


\section{Distribution of Neurons across the Surface of the Human Cerebral Cortex}

Pedro F.M. Ribeiro a , Lissa Ventura a , Léa T. Grinberg ${ }^{b, c}$, Renata E.P. Leite ${ }^{b}$, Renata E.L. Ferretti ${ }^{b}$, José Marcelo Farfel ${ }^{b}$, Wilson Jacob Filho ${ }^{b}$, Suzana Herculano-Houzel ${ }^{a}$

anstituto de Ciências Biomédicas, Universidade Federal do Rio de Janeiro, Rio de Janeiro, ${ }^{b}$ Grupo de Estudos em Envelhecimento Cerebral, Faculdade de Medicina, Universidade de São Paulo, São Paulo, 'Instituto Israelita de Ensino e Pesquisa Albert Einstein, São Paulo, Brazil

(pfmrib@gmail.com)

Brain evolution has often been equated with expansion of the cerebral cortex. Within this structure, a relative expansion of the prefrontal cortex is a candidate basis for the increased cognitive functions of humans. Assuming that the number of brain neurons is a limiting factor in determining cognitive capacity, knowing how neurons are distributed across prefrontal and other regions of the human cerebral cortex is of great importance for deriving conclusions about why our computational abilities differ from those of other species.

Here we examine whether neurons are distributed differently between prefrontal cortex and other areas of the human cerebral cortex, and whether local variations in cortical gyrification are, as traditionally expected, a simple function of local numbers of neurons and/or cortical thickness. We sliced one cerebral cortical hemisphere (female, age 65 years) into a series of $2 \mathrm{~mm}$ coronal sections. After digitalizing the surface of each section for reconstruction, we dissected the cortical gray and white matters of each section and determined the total number of neurons and other cells in them using the Isotropic Fractionator.

We find that the relationship between the volume of the gray matter, $\mathrm{V}_{\mathrm{G}}$, and its number of neurons, $\mathrm{N}_{\mathrm{G}}$, is a shared power function across all cortical sections (including prefrontal cortex) except for the posterior (occipital) cortex, varying with $\mathrm{N}_{\mathrm{G}}{ }^{0.84}$ across the former and with $\mathrm{N}_{\mathrm{G}}{ }^{1.20}$ across the latter. Accordingly, neuronal densities are much larger in the posterior cortex than in any other sections, such that a same $\mathrm{V}_{\mathrm{G}}$ holds a much larger $\mathrm{N}_{\mathrm{G}}$ in occipital compared to other cortical areas.

The local gyrification index (GI) of each section increases as different functions of $\mathrm{N}_{\mathrm{G}}$ in the prefrontal and posterior cortices, with a much larger GI in prefrontal than in posterior cortices for a same $\mathrm{N}_{\mathrm{G}}$. $\mathrm{GI}$ is not significantly correlated with $\mathrm{N}_{\mathrm{G}}$ across the remaining sections. At the same time, we find no correlation between GI and cortical thickness (T) across sections.

In contrast to the different relationships between $\mathrm{V}_{\mathrm{G}}, \mathrm{N}_{\mathrm{G}}$ and GI between the posterior cortex and the other sections, we find a shared relationship between $\mathrm{V}_{\mathrm{G}}$ and its number of other cells, $\mathrm{O}_{\mathrm{G}}$; between the volume of the white matter, $\mathrm{V}_{\mathrm{W}}$, and $\mathrm{O}_{\mathrm{W}}$; and between the ratio $\mathrm{O}_{\mathrm{G}} / \mathrm{N}_{\mathrm{G}}$ and the density of neurons across all sections.

The human prefrontal cortex is thus similar to other cortical regions in its distribution of neurons in the gray matter; the exception to the rule, instead, is the posterior cortex. The fact that $\mathrm{GI}$ is not a single, homogeneous function of $\mathrm{T}$ or $\mathrm{N}_{\mathrm{G}}$ implies that gyrification is not a simple result of the lateral expansion of the gray matter. Importantly, our findings extend to different regions within the cerebral cortex our previous observation across species that while neuronal scaling rules are free to vary, non-neuronal scaling rules are shared, and thus possibly more constrained in evolution.

\section{Comparative Analysis of Environmental Complexity on Adult Hippocampal Neurogenesis in Wild-Living Animals}

Nina Patzke, Amadi O. Ihunwo, Paul R. Manger

School of Anatomical Sciences, Faculty of Health Sciences, University of the Witwatersrand, Johannesburg, South Africa (Nina.Patzke@wits.ac.za)

The hippocampus is crucial for learning and memory formation and is one of the areas where new neurons are generated throughout life. These newborn neurons arise in the subgranular zone of the hippocampal dentate gyrus, mature locally to granular cells and become fully integrated into hippocampal circuitry. Adult hippocampal neurogenesis (AHN) has been demonstrated to be influenced by the complexity of the environment. Specifically an enriched environment was demonstrated to lead to a robust increase in newly generated neurons; however, since most of the studies on adult hippocampal neurogenesis were carried out on laboratory animals, kept under artificial conditions, even in the enriched environment, it is difficult to estimate the functional importance of adult hippocampal neurogenesis from these results.

In order to understand the effect of environment on adult hippocampal neurogenesis we have examined, with immunohistochemistry for doublecortin, the hippocampi of several mammalian species that have fully aquatic or semi-aqautic lifestyles and compared them to their close terrestrial relatives. Our preliminary data led to the conclusion that AHN is strongly related to the environmental complexity inhabited by the animal.

This study was prompted by our observations on the giant otter shrew (Potomogale velox) - a semi-aquatic Afrotherian mammal that revealed extensive AHN; whereas a complete lack of AHN was observed in the Florida manatee (Trichechus manatus) - a fully aquatic member of the Afrotheria. Studies on the golden mole (Amblystomus hottentotus) and the African elephant (Loxodonta africana), other Afrotheria, show extents of AHN that would be considered typical for a terrestrial mammal. We have also studied AHN in the harbour porpoise (Phocoena phocoena) and minke whale (Balaenoptera acutorostra$t a)$, two cetacean species that are fully aquatic members of the Cetartiodactyla order, and interestingly in both these species we could not identify any AHN. AHN in the giraffe and blesbok, two terrestrial members of the Cetartiodactyla, was significant in both animals. To establish the cetacean or aquatic specificity of the lack of adult hippocampal neurogenesis in fully aquatic mammals we plan to examine AHN in several more terrestrial Cetartiodactyla. Lastly, we have also examined the hippocampus of the harbour seal (Phoca vitulina), a semiaquatic member of the carnivores and have observed very high $\mathrm{AHN}$ in this species. Further studies on several species of carnivore, including another semi-aquatic species the Asian small- 
clawed otter, and several terrestrial species are planned to examine AHN to determine whether our previous observations are supported.

Taken together, our current conclusion is that as the fully aquatic environment is, in relative terms, information sparse and AHN may not be required for normal life, whereas, in species that are semi-aquatic, i.e. inhabiting two different environments, there is a strong need for significant AHN as they will encounter several different and varied environmental conditions.

\section{Re-Evaluation of the Classification of D1 Dopamine Receptor Genes in Vertebrates}

Kei Yamamoto, Olivier Mirabeau, Charlotte Bureau, Maryline Blin, Philippe Vernier

Neurobiology and Development (UPR3294), CNRS, Gif-sur-Yvette, France

(kei.yamamoto@inaf.cnrs-gif.fr)

Dopamine neurotransmission is important for various brain functions such as motor programming, motivational process, or learning and memory. The D1 class of dopamine receptors is molecularly characterized by intronless genes and by the ability to activate adenylyl cyclase. While mammals possess only two D1 receptor subtypes, D1A (D1) and D1B (D5), additional subtypes have been found in non-mammalian vertebrates. The third subtype named D1C was originally found in Xenopus [Sugamori et al. 1994], and later found in several teleost fishes. A fourth subtype of $\mathrm{D} 1$ receptor named D1D was isolated from the chick genome, which was considered to be a separate subtype based on its unique pharmacological profile, as well as on its relatively low sequence identity with the D1C receptor [Demchyshyn et al. 1995]. Finally, a supernumerary D1X receptor sequence, yet pharmacologically uncharacterized, was found in the carp retina [Hirano et al., 1998].

The phylogenetic analysis of the D1 receptor class and a scenario of its evolution have been previously presented, in which D1D and D1X receptor sequences were considered to be an outgroup of the D1A, B, and $\mathrm{C}$ receptor sequences [Callier et al. 2003; Le Crom et al. 2003; Le Crom et al. 2004]. Here we propose a new evolutionary view of the D1 class receptors in jawed vertebrates, based on phylogenetic analysis from a wider range of vertebrate species, as well as detailed gene syntenies. Firstly, we revealed that D1C and D1D are encoded by orthologous genes. We thus propose to rename the avian D1D to D1C. The gene synteny strongly suggests that $D 1 A, D 1 B$, and $D 1 C$ genes were present in the stem jawed vertebrates, and that mammals secondarily lost the D1C receptor gene. Secondly, some teleosts possess two copies of each subtype, as a consequence of the additional whole genome duplication. The Cypriniform $D 1 X$ sequence is likely to be a teleost-specific paralogue of the $D 1 B$ sequences. Thirdly, several species (e.g. zebrafish, lizard, and turkey) possess an additional D1-like gene, which is likely to form another group of orthologous. We propose to name it D1E. Thus, ancestral jawed vertebrates were likely to possess four classes of D1 receptor genes $D 1 A, D 1 B(X), D 1 C(D)$, and $D 1 E$. Then $D 1 C$ has been secondarily lost in the mammalian lineage, while $D 1 E$ has lost in several lineages of modern vertebrates independently.

The D1A receptor is the best conserved subtype in jawed vertebrates, as is its expression in the striatum and cell functions. In contrast, sauropsid D1Cs have rapidly diverged, to the point they were misidentified as D1D. In birds, D1C is selectively expressed in the pallium, where effects of dopamine are often comparable to those in the mammalian cortex. It would be interesting to know whether D1C plays an role specific to birds, or the functions have been substituted by D1A or D1B in mammals following its disappearance.

\section{References}

Callier S, Snapyan M, Le Crom S, Prou D, Vincent JD, Vernier P (2003) Evolution and cell biology of dopamine receptors in vertebrates. Biol Cell 95:489-502.

Demchyshyn LL, Sugamori KS, Lee FJ, Hamadanizadeh SA, Niznik HB (1995) The dopamine D1D receptor. Cloning and characterization of three pharmacologically distinct D1-like receptors from Gallus domesticus. J Biol Chem 270:4005-4012.

Hirano J, Archer SN, Djamgoz MB (1998) Dopamine receptor subtypes expressed in vertebrate (carp and eel) retinae: cloning, sequencing and comparison of five D1-like and three D2-like receptors. Receptor Channels 5:387-404.

Le Crom S, Kapsimali M, Barome PO, Vernier P (2003) Dopamine receptors for every species: gene duplications and functional diversification in Craniates. J Struct Funct Genomics 3:161-176.

Le Crom S, Sugamori KS, Sidhu A, Niznik HB, Vernier P (2004) Delineation of the conserved functional properties of D1A, D1B and D1C dopamine receptor subtypes in vertebrates. Biol Cell 96:383-394.

Sugamori KS, Demchyshyn LL, Chung M, Niznik HB (1994) D1A, D1B, and D1C dopamine receptors from Xenopus laevis. Proc Natl Acad Sci USA 91:10536-10540.

\section{Selective, Parallel Expansion of the Voltage-Gated Sodium Channel Family in Teleosts and Tetrapods Coincides with the Evolution of Forebrain Complexity}

\section{Harold H. Zakon}

Sections of Neurobiology and Integrative Biology, The University of Texas, Austin, Tex., USA

(h.zakon@austin.utexas.edu)

Mammals have ten voltage-dependent sodium (Nav) channel genes. Nav channels are expressed in different cell types with different sub-cellular distributions and are critical for many aspects of neuronal processing. The last common ancestor of teleosts and tetrapods had four Nav channel genes, presumably on four different chromosomes. In the lineage leading to mammals, a series of tandem duplications on two of these chromosomes more than doubled the number of Nav channel genes. It is unknown when these duplications occurred and whether they occurred against a backdrop of duplication of flanking genes or as an expansion of ion channel genes in general. We estimated key dates of the Nav channel gene family expansion by phylogenetic analysis using 
teleost, elasmobranch, lungfish, amphibian, avian, lizard, and mammalian Nav channel sequences, as well as chromosomal synteny for tetrapod genes. We tested, and exclude, the null hypothesis that Nav channel genes reside in regions of chromosomes prone to duplication by demonstrating the lack of duplication or duplicate retention of surrounding genes. We also find no comparable expansion in other depolarizing (calcium, transient receptor potential [TRP], hyperpolarization-activated cyclic nucleotide-gated $[\mathrm{HCN}]$ ) or hyperpolarizing (voltage-gated $[\mathrm{Kv}]$, ether-a-go-go-related [ERG], or calcium-activated potassium [slo] ion channel gene families of tetrapods following the teleosttetrapod divergence. We posit a specific expansion of the Nav channel gene family in the Devonian and Carboniferous periods when tetrapods evolved, diversified, and invaded the terrestrial habitat. During this time, the amniote forebrain evolved greater anatomical complexity and novel tactile sensory receptors appeared. The duplication of Nav channel genes allowed for greater regional specialization in Nav channel expression, variation in subcellular localization, and enhanced processing of somatosensory input. 